\title{
The reverse Silfverskiöld test in Achilles tendon rupture
}

\author{
D. Joshua Mayich, MD; ${ }^{*}$ AlastairYounger, $\mathrm{MD}_{;}^{\dagger}$ Fabian Krause, $\mathrm{MD}^{\ddagger}$
}

\section{INTRODUCTION}

Achilles tendon rupture is a relatively common injury, with a mean Canadian annual incidence of 8.3 ruptures per 100000 people. ${ }^{1}$ Achilles tendon rupture typically occurs in the third to fifth decade of life, although it can occur anywhere within the second and eighth decades of life. ${ }^{2}$ This injury is believed to be due to a combination of chronic disease in the tendon substance, and a profound and quickly applied dorsiflexion force on the foot. ${ }^{1-5}$ Although uncommon, this injury may present in a delayed or "neglected" manner, therefore clouding the presentation. ${ }^{4}$

There are several clinical tests to facilitate in the diagnosis of Achilles tendon rupture. However, indications for operative versus conservative management can sometimes be difficult to define. For example, visual inspection for a gap in the tendon substance and palpation of the Achilles tendon are widely used techniques. However, these can be unreliable, and thus nondiagnostic, in the context of acute swelling and bruising. The Thompson test, which was first described in $1962,{ }^{6}$ is a widely taught and used method of clinically evaluating the competency of the Achilles tendon. A single-leg heel raise is also a common diagnostic text in suspected Achilles tendon rupture, but is frequently impossible to perform owing to pain.

Although Achilles tendon rupture is generally considered a clinical diagnosis, difficulty can occur when the physical examination is equivocal. In such situations, diagnostic imaging can be beneficial. ${ }^{3}$ Further difficulty can arise when an imaged tendon is reported to show a "partial" or "incomplete" tear. In this situation, the clinician must rely on clinical tests to determine whether the plantaris muscle or a tendon overlap- ping the rupture site is responsible for an incorrect diagnosis on imaging, and that the Achilles tendon is no longer intact.

In the setting of a subacute or chronic rupture, scar formation may masquerade as functional tendon tissue on ultrasonography or magnetic resonance imaging. Finally if "no rupture" is diagnosed, it remains possible that a partial tear has occurred, functionally lengthening the Achilles tendon and resulting in a mechanical disadvantage to the pull of the triceps surae muscle group.

\section{THE REVERSE SILFVERSKIÖLD TEST}

The Silfverskiöld test was originally developed to determine whether an Achilles tendon was shortened. ${ }^{7}$ It was apparent to us that it could also be used in reverse if the Achilles tendon was functionally too long. At the BC Foot and Ankle Clinic, we have used this reverse test to determine in equivocal cases whether surgical intervention was required to repair or shorten the tendon.

\section{Technique}

The patient is seated and both legs are exposed. First, the uninjured ankle is examined for passive dorsiflexion in both the extended knee position and the flexed knee position. The relative excursion of the uninjured Achilles tendon is thus determined, providing the clinician with a comparison (Fig. 1). A goniometer can be used to more accurately determine the range of motion (ROM) achievable in both the extended and flexed knee positions.

Following this, the relative ROM of the injured leg is

From the *Department of Orthopaedic Surgery, Queen's University, Kingston, Ont., the †BC Foot and Ankle Clinic, University of British Columbia, Vancouver, BC, and the ¥Department of Orthopaedic Surgery, University of Berne, Berne, Switzerland

Submitted Aug. 2, 2008; Accepted Sep. 16, 2008

This article has not been peer reviewed.

CJEM 2009;11(3):242-3 
examined in a similar fashion. Specifically, the achievable dorsiflexion in the extended knee position is examined (Fig. 2). The range of dorsiflexion with the ex-

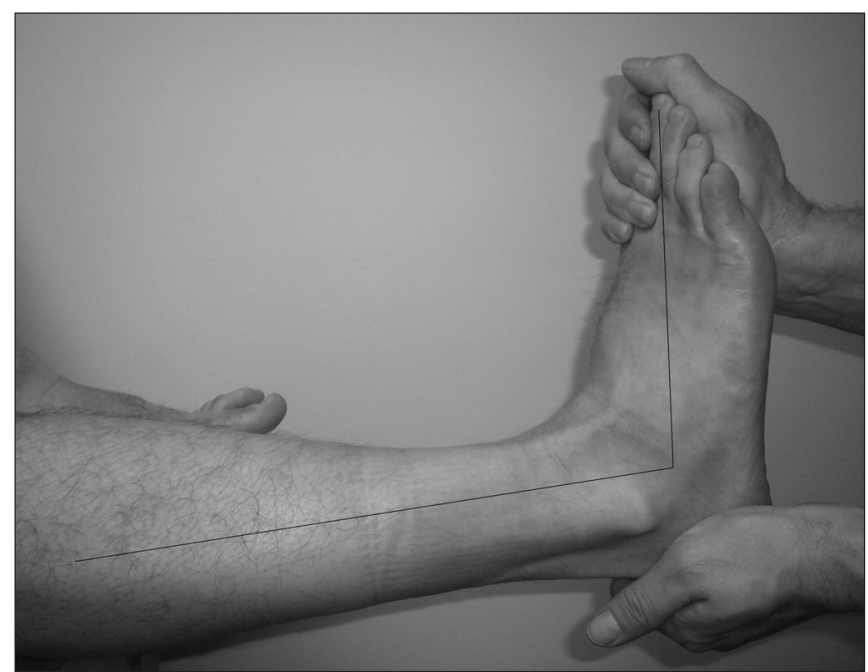

Fig. 1. The reverse Silfverskiöld test on the uninjured side reveals neutral dorsiflexion.

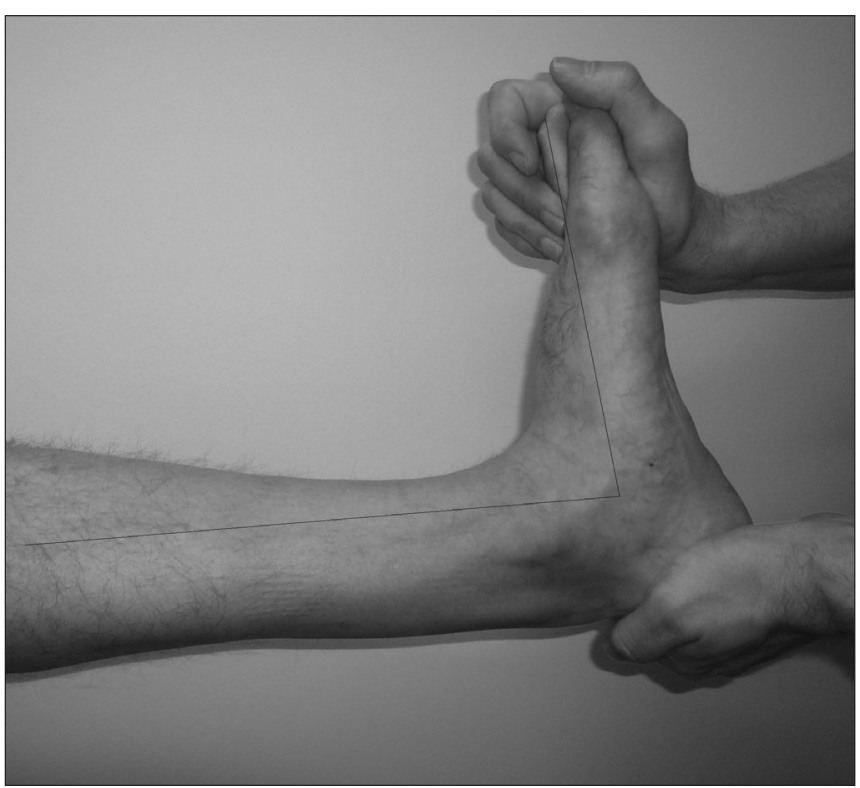

Fig. 2. The reverse Silfverskiöld test on the injured side reveals, by comparison, roughly 10 degrees of dorsiflexion. tended knee is solely restricted by the Achilles tendon in this position.

A notable difference between the injured and uninjured side in dorsiflexion in the extended knee position reflects the functional excursion of the Achilles tendon. This, in turn, indicates the competency of the tendon itself. Greater than expected dorisflexor ROM in the extended knee position can indicate the presence of a tear in the tendon or, in the setting of a delayed presentation, may indicate that the tendon has healed and lengthened.

\section{Competing interests: None declared.}

Keywords: Achilles tendon rupture, Achilles tendon tear, gastrocnemius, triceps suri, Silfverskiöld

\section{REFERENCES}

1. Suchak AA, Bostick G, Reid D, et al. The incidence of Achilles tendon ruptures in Edmonton, Canada. Foot Ankle Int 2005;26:932-6.

2. Chiodo CP, Wilson MG. Current concepts review: acute ruptures of the achilles tendon. Foot Ankle Int 2006;27:305-13.

3. Coughlin MJ, Mann RA, Saltzman CL. Surgery of the foot and ankle. 8th ed. Philadelphia (PA): Mosby Elsevier; 2007.

4. Leslie HD, Edwards WH. Neglected ruptures of the Achilles tendon. Foot Ankle Clin 2005;10:357-70.

5. Popovic N, Lemaire R. A diagnosis and treatment of acute ruptures of the Achilles tendon. Current concepts review. Acta Orthop Belg 1999;65:458-71.

6. Thompson TC. A test for rupture of the tendo achillis. Acta Orthop Scand 1962;32:461-5.

7. Silfverskiold N. Reduction of the uncrossed two-joints muscles of the leg to one-joint muscles in spastic conditions. Acta Chir Scand 1924;56:315-28.

Correspondence to: Dr. Alastair Younger, Clinical Associate Professor, Department of Orthopaedic Surgery, University of British Columbia, Suite 560, 1144 Burrard St., Vancouver BC V6Z 2A5; asyounger @telus.net 